\title{
Vacunas para prevenir la infección neumocócica en adultos
}

\section{Objetivo}

Evaluar la efectividad de la vacuna de polisacárido neumocócico para prevenir la enfermedad o muerte en adultos.

\section{Estrategia de búsqueda}

Se identificaron ensayos del Registro Cochrane de Ensayos Controlados, MEDLINE (1966-2003) y EMBASE (1974 -2003); se contactó a los fabricantes de la vacuna y a los autores de los estudios identificados para mejorar la sensibilidad.

\section{Criterios de selección}

Estudios prospectivos, aleatorizados o cuasialeatorizados*, que comparan las vacunas neumocócicas con placebo, vacunas control (antigripal) o ninguna intervención; y estudios de casos y controles no aleatorizados, con la idea de obtener el poder suficiente para evaluar el punto final enfermedad neumocóccica invasiva, de muy baja incidencia.

\section{Recopilación y análisis de datos}

Dos revisores evaluaron la calidad de los ensayos. Para los aleatorizados se utilizó el score de Jadad*

\section{Resultados principales}

Los resultados combinados de los estudios aleatorizados no demuestran si la vacuna de polisacárido neumocócico es efectiva para la prevención de la neumonía (OR 0,77; IC95\% 0,58 a 1,02) o la muerte (OR 0,90; IC 0,76 a 1,07). A pesar de los datos alentadores de algunos ensayos muy antiguos, la combinación de ensayos publicados desde 1977 en adelante sugiere que no hay un efecto en la prevención de neumonía (OR 0,96; IC95\% 0,80 a 1,15) ni en la mortalidad por neumonía (OR 0,98; IC $95 \%$ 0,88 a 1,09).
Los datos disponibles no permiten distinguir si esta heterogeneidad en los resultados se debe a mejoras en la metodología de los trabajos, a diferencias en el lugar de los ensayos o a una pérdida de eficacia real con el tiempo. Esto se debe a que los ensayos antiguos, de peor calidad metodológica, fueron realizados en poblaciones saludables de alto riesgo, donde se espera que el beneficio sea mayor.

Los estudios de casos y controles muestran una eficacia significativa en la prevención de la enfermedad neumocócica invasiva, especialmente en mayores de 55 años: OR 0,47 (IC95\% 0,37 a $0,59)$, lo que corresponde a una eficacia del $53 \%$.

\section{Conclusiones del revisor}

Aunque las vacunas de polisacárido neumocócico no parecen reducir la incidencia de neumonía o muerte en adultos con o sin enfermedad crónica o en personas de edad avanzada (55 años o más), la evidencia de estudios no aleatorizados sugiere que las vacunas son efectivas en la reducción de la incidencia del resultado más específico, la enfermedad neumocócica invasiva, en adultos y en personas de edad avanzada inmunocompetentes ( 55 años o más). Los datos de vigilancia epidemiológica sugieren que las tasas de infección varían ampliamente entre países y también dentro de cada país, pero la cifra típica en los países desarrollados es $0,01 \%$, ó 10 cada 100000 por año. Por lo tanto, la eficacia del $50 \%$ corresponde al número necesario a tratar (NNT) de 20000 vacunas por infección que se evita y quizás 50000 por muerte que se evita.

Fuente de financiamiento: Ninguna

\section{Comentario}

Las enfermedades causadas por el Streptococcus pneumoniae (Sp) continúan siendo causa de morbilidad y mortalidad considerable en todo el mundo. Se han reportado tasas de infección por $\mathrm{Sp}$ del 30 al $50 \%$ de las neumonías agudas de la comunidad (NAC), siendo la mortalidad cercana al $25 \%$. Esta no ha variado desde hace 40 años ${ }^{1-2}$.

La enfermedad se produce con mayor frecuencia en personas de edad avanzada (> 55 años), con enfermedades crónicas o inmunocompromiso. Además de NAC, el Sp causa diversas infecciones sistémicas serias como meningitis y bacteriemia, de alta mortalidad.

En cuanto a la diversidad de Sp es importante mencionar que una revisión $C D C$ de EE.UU. mostró que los no vacunados con infecciones neumocócicas estaban infectados en el $67 \%$ de los casos por serotipos incluidos en la vacuna de 14 valencias y en el $88 \%$ de los casos con serotipos de la vacuna de 23 valencias $^{3}$.

Por lo mencionado anteriormente y porque la resistencia a los antibióticos es una amenaza importante para el éxito del tratamiento de las infecciones ${ }^{4-5}$, existe necesidad urgente de saber si las vacunas neumocócicas son efectivas en todas las poblaciones, o si solamente se beneficiarán algunos grupos. Durante más de 50 años se han desarrollado nuevas vacunas de polisacárido neumocócico pero la controversia acerca de la efectividad de las mismas continúa ya que existen múltiples metaanálisis con resultados dispares.
Esto se refleja en la variabilidad existente entre las recomendaciones de diversos países $^{2-6}$. La Fuerza de Tareas de Estados Unidos la recomienda para todos los mayores de 65 años o en pacientes con inmunocompromiso o diversas enfermedades crónicas. La Fuerza de Tareas Canadiense, en cambio, basándose en el metaanálisis de Fine no recomienda la vacunación para los pacientes con enfermedades crónicas. En Inglaterra se está planificando hacerla disponible para todos los mayores de 65 pero la recomendación actual es para los mayores de 80 años.

Este metaanálisis intenta arrojar claridad en cuanto a los resultados divergentes de las revisiones anteriores, poniendo de manifiesto la regular calidad y heterogeneidad de la evidencia existente. En este sentido se incluyeron estudios realizados en épocas en donde las posibilidades de tratamiento de la NAC eran muy limitadas y la mortalidad mucho mayor (1947), de esta manera los estudios antiguos muestran una significativa disminución de la misma con la vacuna mientras que estudios más recientes no logran demostrar el mismo efecto.

\section{Conclusión del comentador}

Según la mejor evidencia disponible, las vacunas antineumocóccicas han demostrado regular efectividad para prevenir NAC y muerte, pero disminuirían la incidencia de infecciones sistémicas. Es importante destacar que la investigación en este terreno no debería ser abandonada.

Dra. Alejandrina Losasso. [ [ Unidad de Medicina Familiar y Preventiva. Hospital Italiano de Buenos Aires.]

* ver glosario

Losasso A. Vacunas para prevenir la infección neumocócica en adultos. Evid. actual. práct. ambul 2004;7:101. Comentado de: Dear K, Holden J, Andrews R, Tatham D. Vaccines for preventing pneumococcal infection in adults. Cochrane Database Syst Rev. 2003;(4):CD000422.

\section{Bibliografía}

1.Hirschmann JV, Lipsky BA. The Pneumococcal Vaccine After 15 Years of Use. Archives of Internal Medicine 1994;154:373-7.

2.Butler JC, Breiman RF, Campbell JF, Lipman HB, Broome CV, Facklam RR. Pneumococcal polysaccharide vaccine efficacy. An evaluation of current recommendations. JAMA 1993;270(15):1826-31.

3.Calendario de vacunaciones recomendadas para adultos por grupo de edad y condiciones médicas, EEUU, 2003-2004, Departamento de Salud y Servicios Humanos Centros para el Control y la Prevención de Enfermedades.

4.Pallares R, Linares J, Vadillo M, Cabellos C, Manresa F, Viladrich PF et al. Resistance to Penicillin and Cephalosporin and Mortality from Severe Pneumococcal Pneumonia in Barcelona, Spain. New England Journal of Medicine 1995;333:474-80.

5.Reacher MH, Shah A, Livermore DM, Wale MCJ, Graham C, Johnson AP et al. Bacteraemia and antibiotic resistance of its pathogens reported in England and Wales between 1990 and 1998: trend analysis. BMJ 2000;320:213-6.

6.WHO. Pneumococcal vaccines. Weekly Epidemiology Record 1999;74(23):177-83 